\title{
Double differential light charged particle emission cross sections for some structural fusion materials
}

\author{
Ismail Hakki Sarpün ${ }^{1}$, Abdullah Aydın², and Eyyup Tel ${ }^{3}$ \\ 1 Afyon Kocatepe University, Department of Physics, Afyonkarahisar, Turkey \\ 2 Kirikkale University, Department of Physics, Kirikkale, Turkey \\ 3 Osmaniye Korkut Ata University, Department of Physics, Osmaniye, Turkey
}

\begin{abstract}
In fusion reactors, neutron induced radioactivity strongly depends on the irradiated material. So, a proper selection of structural materials will have been limited the radioactive inventory in a fusion reactor. First-wall and blanket components have high radioactivity concentration due to being the most fluxexposed structures. The main objective of fusion structural material research is the development and selection of materials for reactor components with good thermo-mechanical and physical properties, coupled with low-activation characteristics. Double differential light charged particle emission cross section, which is a fundamental data to determine nuclear heating and material damages in structural fusion material research, for some elements target nuclei have been calculated by the TALYS 1.8 nuclear reaction code at $14-15 \mathrm{MeV}$ neutron incident energy and compared with available experimental data in EXFOR library. Direct, compound and pre-equilibrium reaction contribution have been theoretically calculated and dominant contribution have been determined for each emission of proton, deuteron and alpha particle.
\end{abstract}

\section{Introduction}

Fusion reactors need proper structural materials to become an efficient source of energy, especially if the promise of a green machine is to be maintained. Reactor components, such as first wall, divertor and blanket, are subjected not only to the high-energy neutron impact, but also to strong mechanical, heat and electromagnetic loadings. Activation characteristics of candidate materials are important in classification as low activation elements $(\mathrm{C}, \mathrm{Si}, \mathrm{Ti}, \mathrm{Fe}, \mathrm{Cr}$ and $\mathrm{V}$ ) and as high activation materials ( $\mathrm{Al}, \mathrm{Ni}, \mathrm{Ag}, \mathrm{Co}$ and $\mathrm{Nb}$ ) [1-4]. The choice of the materials depends not only on mechanical properties, compatibility with other materials and irradiation performance, but also on their radiological properties [5-7]. The aim of the fusion structural material research studies is to develop fusion reactor structural materials with good thermo-mechanical and physical properties coupled with low-activation characteristics.

In a fusion reactor neutrons carry most of the energy produced by the $\mathrm{D}-\mathrm{T}$ fusion reaction. Those neutrons lose their energy by interacting with reactor devices such as the blanket through various nuclear reactions and the heat produced by these nuclear reactions is called "nuclear heating" and is estimated by a simulation calculation in a reactor design. One of the essential data for simulation calculation is the energy and angular distributions of charged particles emitted by a nuclear reaction. These distributions are called "double differential cross section (DDX) data". The DDX of light charged particle emission have been investigated by several researches both experimentally [8-16] and theoretically [17-32].

In this study, the DDX for the emission of alpha and proton induced by $14.8 \mathrm{MeV}$ neutrons from ${ }^{50,52} \mathrm{Cr}$ and ${ }_{58,60} \mathrm{Ni}$, and by $15.0 \mathrm{MeV}$ neutrons for ${ }^{46,48} \mathrm{Ti}$ target nuclei have been calculated using TALYS 1.8 code and the results have been compared with the available experimental data in literature.

\section{Calculation methods}

TALYS 1.8 code has been used in DDX calculation according to pre-equilibrium exciton model, direct model and compound nucleus formation [33]. The TALYS 1.8 code is able to analyze and predict nuclear reactions induced by up to $1 \mathrm{GeV}$ light particles $(\mathrm{A} \leq 4)$ for target nuclei heavier than lithium [34]. The two-component exciton model developed by Kalbach [35] was used for calculating the pre-equilibrium contribution while the compound nucleus was calculated by the HauserFeshbach model [36]. Direct reaction calculation is obtained via the ECIS-97 [37] code which is implemented as a subroutine in TALYS.

\section{Results and conclusions}

The DDX $\left(\mathrm{d}^{2} \sigma / \mathrm{d} \Omega\right.$.dE) for the emission of $\mathrm{p}$ and $\alpha$ particles at emission angles $30^{\circ}, 45^{\circ}, 90^{\circ}$ and $135^{\circ}$ for ${ }^{50,52} \mathrm{Cr}$ and ${ }^{58,60} \mathrm{Ni}$ for incident neutron energy of $14.8 \mathrm{MeV}$, and $45^{\circ}, 60^{\circ}, 75^{\circ}$ and $135^{\circ}$ for ${ }^{46,48} \mathrm{Ti}$ target nuclei for incident neutron energy of $15.0 \mathrm{MeV}$ have been calculated using TALYS 1.8 nuclear reaction code. The calculated energy distribution of the emitted light charged particles has been compared with available experimental data of Grimes et al. [38] taken form the EXFOR [39] nuclear data library.

There is a good agreement between the DDX results theoretically calculated using TALYS 1.8 and the experimental data of Grimes et al. [38]. Further, the 


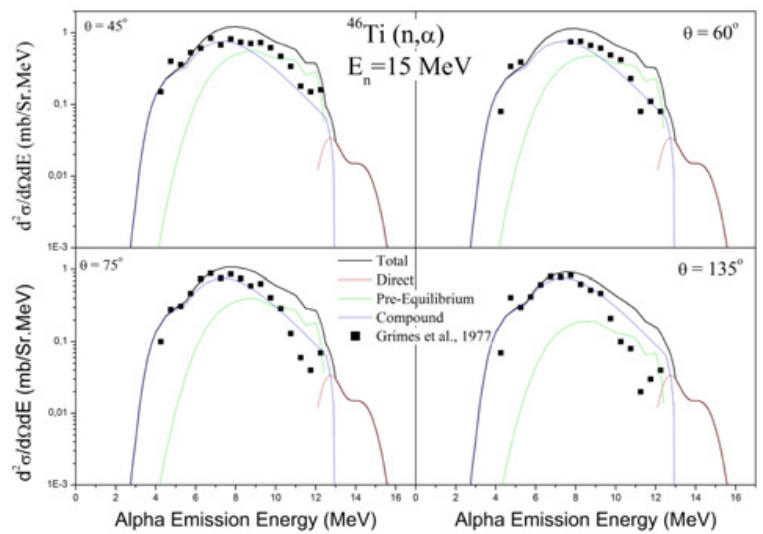

Figure 1. The comparison of calculated DDX of $(n, \alpha)$ reaction on ${ }^{46} \mathrm{Ti}$ with the experimental data of Grimes [38] available in EXFOR [39].

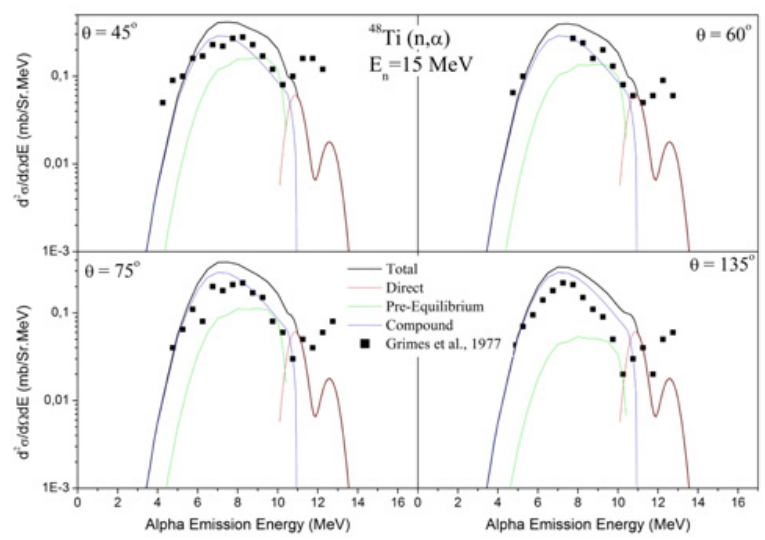

Figure 2. The comparison of calculated DDX of $(\mathrm{n}, \alpha)$ reaction on ${ }^{48} \mathrm{Ti}$ with the experimental data of Grimes [38].

variation in the DDX with the emission angle indicates that the emission of alpha and proton shows similar distribution to the isotropic distribution.

The theoretically calculated and experimental DDX of alpha emission for (n, $\alpha$ ) reactions on ${ }^{46} \mathrm{Ti},{ }^{48} \mathrm{Ti},{ }^{50} \mathrm{Cr},{ }^{52} \mathrm{Cr}$, ${ }^{58} \mathrm{Ni}$ and ${ }^{60} \mathrm{Ni}$ target nuclei at incident neutron energy of 14.8 or $15.0 \mathrm{MeV}$ are shown in Figs. 1-6 respectively.

Theoretically calculated DDX for the emission of alpha particles from ${ }^{46} \mathrm{Ti}(\mathrm{n}, \alpha)$ and ${ }^{48} \mathrm{Ti}(\mathrm{n}, \alpha)$ reactions at 15.0 MeV (Figs. 1, 2) shows that the alpha particles up to the most probable energies are emitted predominantly through the compound nucleus formation whereas the higher energy alpha particles are emitted through the preequilibrium or the direct reaction. While the compound contribution is dominant up to $8 \mathrm{MeV}$ and the preequilibrium process is in the range of $8-12 \mathrm{MeV}$ at $45^{\circ}$ alpha emission angle, the emission probabilities are very low for energetic alpha particles at $135^{\circ}$ emission angle.

The calculated and experimental DDX of alpha emission for ${ }^{50} \mathrm{Cr}(\mathrm{n}, \alpha)$ and ${ }^{52} \mathrm{Cr}(\mathrm{n}, \alpha)$ reactions at 14.8 MeV are shown in Figs. 3 and 4, respectively. The compound contribution is more dominant in ${ }^{52} \mathrm{Cr}$ target nucleus than ${ }^{50} \mathrm{Cr}$ and increases with increasing angle for both target nuclei. The pre-equilibrium contribution is dominant in the range of $8-12 \mathrm{MeV}$ at $30^{\circ}$ and $45^{\circ}$ alpha emission angles for ${ }^{50} \mathrm{Cr}$ target nucleus. The higher energy alpha particles are emitted through the direct reaction.

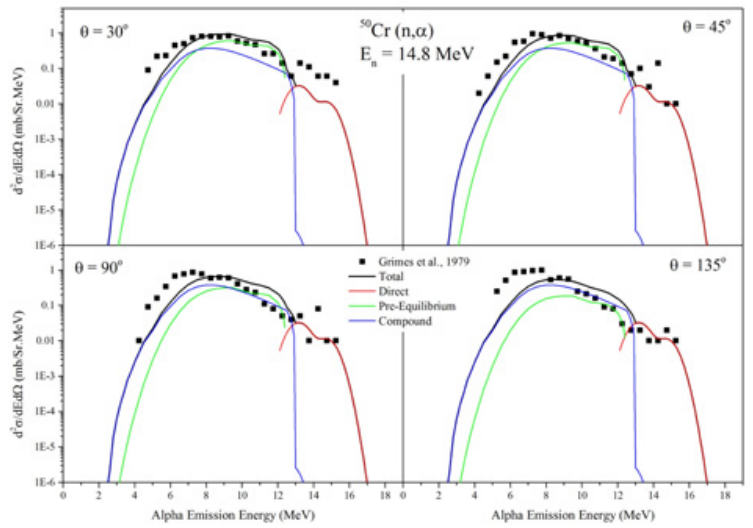

Figure 3. The comparison of calculated DDX of ${ }^{50} \mathrm{Cr}$ target nucleus with the experimental data taken from EXFOR [39].

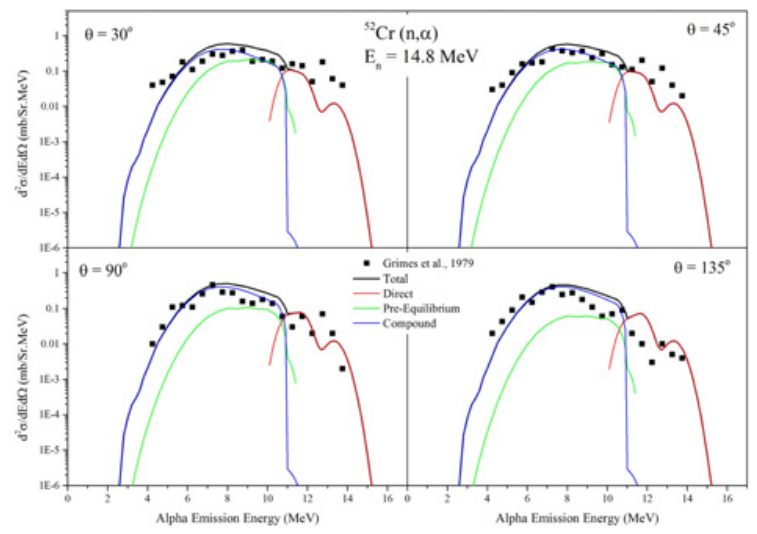

Figure 4. The comparison of calculated DDX of ${ }^{52} \mathrm{Cr}$ target nucleus with the experimental data taken from EXFOR [39].

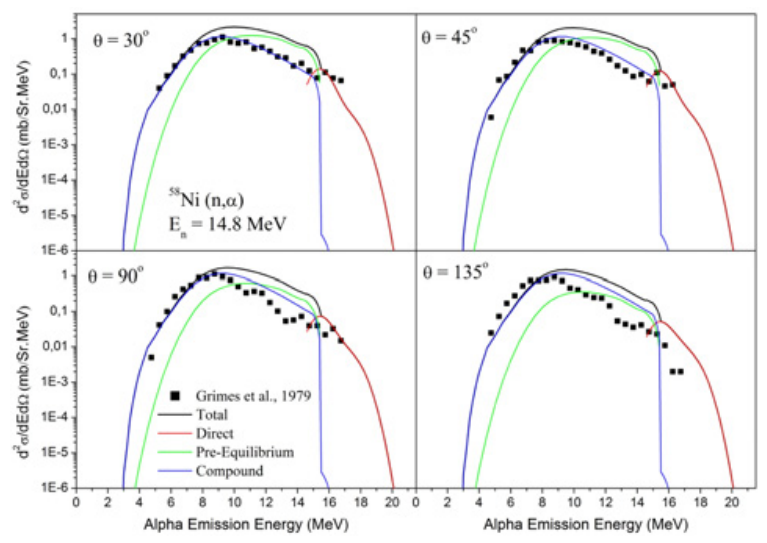

Figure 5. The comparison of calculated DDX of $(n, \alpha)$ reaction on ${ }^{58} \mathrm{Ni}$ with the experimental data of Grimes [38].

The DDX of ${ }^{58} \mathrm{Ni}(\mathrm{n}, \alpha)$ and ${ }^{60} \mathrm{Ni}(\mathrm{n}, \alpha)$ reactions at 14.8 MeV are shown in Figs. 5 and 6, respectively. The compound contribution is dominant in both ${ }^{58} \mathrm{Ni}$ and ${ }^{60} \mathrm{Ni}$ target nuclei and also decreases with increasing angle for both target nuclei. The direct reaction contribution becomes dominant at alpha emission energy $14-15 \mathrm{MeV}$ at $30^{\circ}$ and $45^{\circ}$ alpha emission angles for ${ }^{60} \mathrm{Ni}$ target nucleus and at $16 \mathrm{MeV}$ for all investigated angles for ${ }^{58} \mathrm{Ni}$ nucleus.

The DDX of proton emission are compared with experimental data [38] at incident neutron energy 15.0 MeV as shown in Figs. 7 and 8. The calculated results of DDX for proton emission are mainly from the 


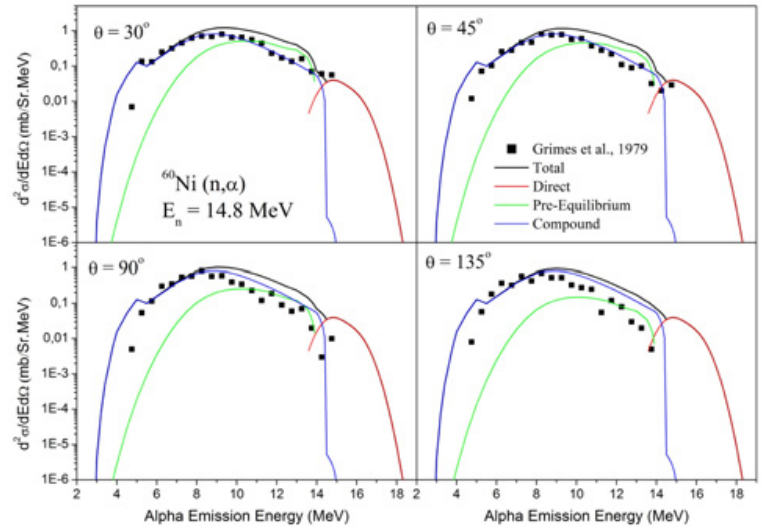

Figure 6. The comparison of calculated double differential alpha emission cross section for ${ }^{60} \mathrm{Ni}$ target nucleus with the experimental data which are taken from EXFOR [39].

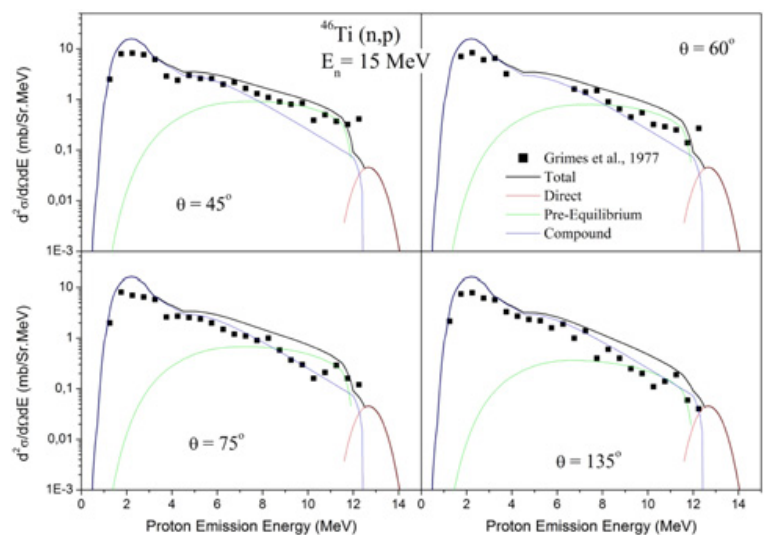

Figure 7. The comparison of calculated DDX of $(n, p)$ reaction on ${ }^{46} \mathrm{Ti}$ with the experimental data reported in literature. Experimental values were taken from EXFOR [39].

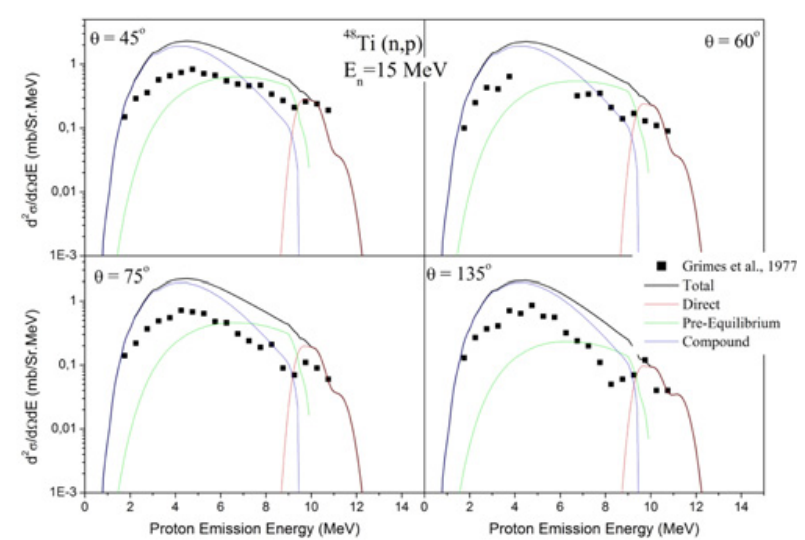

Figure 8. The comparison of DDX of proton emission for ${ }^{48} \mathrm{Ti}$ target nucleus with the experimental data which are taken from EXFOR [39].

contribution of the compound emission. While the preequilibrium emission contribution is observed after $7 \mathrm{MeV}$ proton emission energy for forward emission angles, it is observed after $9 \mathrm{MeV}$ proton emission energy for $135^{\circ}$ backward emission angle. The direct reaction contribution to the proton emission in ${ }^{46} \mathrm{Ti}$ target nucleus is not as much as in ${ }^{48} \mathrm{Ti}$ for the energetic proton emission.

The experimental and calculated DDX of proton emissions are compared at incident neutron energy

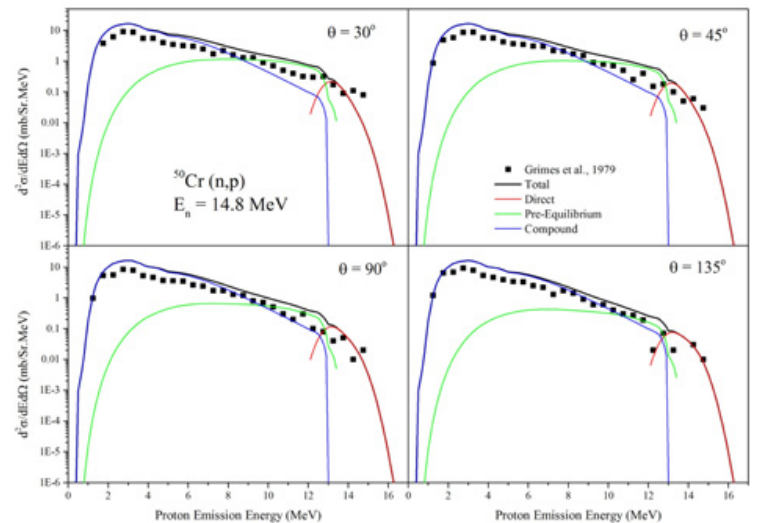

Figure 9. The comparison DDX of proton emission for ${ }^{50} \mathrm{Cr}$ target nucleus with the experimental data which are taken from EXFOR [39].

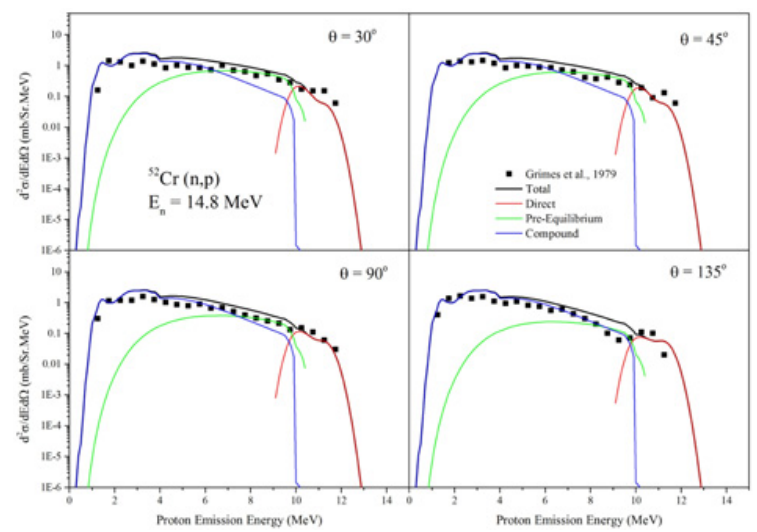

Figure 10. The comparison DDX of proton emission for ${ }^{52} \mathrm{Cr}$ target nucleus with the experimental data which are taken from EXFOR nuclear library [39].

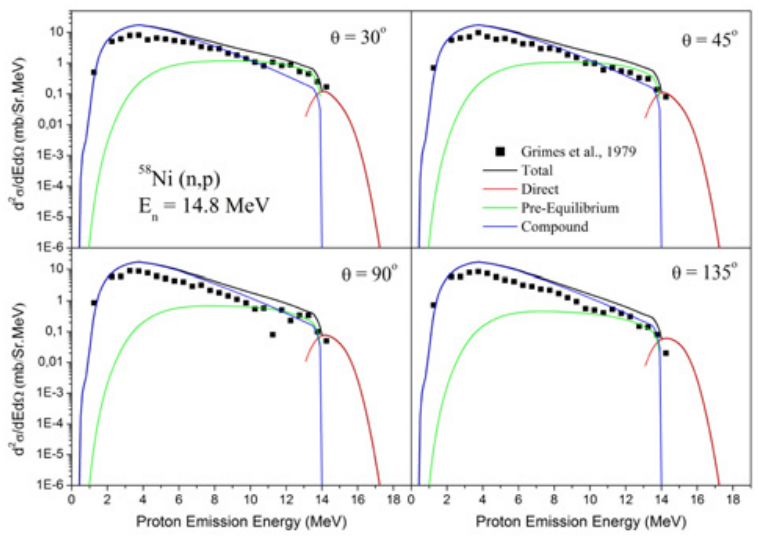

Figure 11. The comparison of DDX of (n,p) reaction on ${ }^{58} \mathrm{Ni}$ with the experimental data taken from EXFOR [39].

14.8 MeV as shown in Figs. 9 and 10. The calculated DDX for proton emissions are mainly from the contribution of the compound emission. While the pre-equilibrium emission contribution is observed after $7 \mathrm{MeV}$ for proton emission energy for ${ }^{50} \mathrm{Cr}$ target nucleus, it is observed after $9 \mathrm{MeV}$ proton emission energy for ${ }^{52} \mathrm{Cr}$ target nucleus.

The DDX of proton emission are shown at neutron incident energy of $14.8 \mathrm{MeV}$ in Figs. 11 and 12 for ${ }^{58} \mathrm{Ni}$ and ${ }^{60} \mathrm{Ni}$, respectively. One can see easily from the Figs. 11 and 12 that the DDX for proton emissions are mainly the 


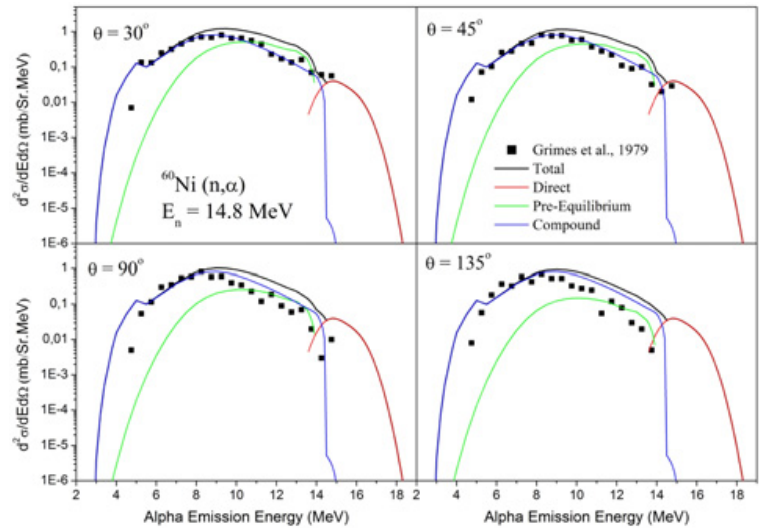

Figure 12. The comparison of DDX of proton emission for ${ }^{60} \mathrm{Ni}$ target nucleus with the experimental data taken from EXFOR [39].

contribution of the compound emission. While the direct emission contribution is observed after $12 \mathrm{MeV}$ for proton emission energy for ${ }^{60} \mathrm{Ni}$ target nucleus, it is not directly observed for ${ }^{58} \mathrm{Ni}$ target nucleus.

\section{Conclusion}

The compound, direct and pre-equilibrium emissions complete the description of the light charged particle emission over the full energy range. TALYS calculations agree quite well with the experimental data of Grimes et al. [38] for proton and alpha emission. The graphs show that for almost all light charged particles emission, compound contribution is dominant. While a strong component from the pre-equilibrium process is present at low emission energy, contribution of direct emission becomes dominant at higher energy for proton emission. In addition, the direct emission contribution arises from the decay of residual nuclei as known. The shapes of the DDX distribution show that nuclear reaction models require a good description of the energy-differential cross sections.

This work is supported by the Afyon Kocatepe University's Scientific Research Office (BAPK) Contract No. 16.KARIYER.34.

\section{References}

[1] E.E. Bloom et al., J. Nucl. Mater. 17, 122-123 (1984)

[2] M. Victoria et al., Nucl. Fusion 41(8), 1047 (2001)

[3] P.M. Raole et al., Trans. IIM 62, 2-105 (2009)
[4] R.J. Kurtz et al., J. Nucl. Mater. 411, 386-388 (2009)

[5] D.R. Harries et al., J. Nucl. Mater. 92, 191-194 (1992)

[6] A. Möslang et al., Int. J. Mater. Res. 99, 1045 (2008)

[7] S.J. Zinkle, J.T. Busby, Mater. Today 12, 12 (2009)

[8] T. Sanami et al., JAERI Conferences Proceedings 008, 96, 231 (1995)

[9] K. Kondo et al., Fusion Eng. Des. 81, 1527-1533 (2006)

[10] B. Lalremruata et al., Nucl. Phys. A 821, 23 (2009)

[11] G.F. Thomas, S.J. Brereton, J. Fusion Energ. 4, 27 (1985)

[12] D.W. Kneff et al., Nucl. Sci. Eng. 92, 491 (1986)

[13] A. Kumar et al., J. Fusion Energ. 8, 107 (1989)

[14] A.A. Cowley et al., Phys. Rev. C 43, 678 (1991)

[15] I.M. Kokooo et al., Nucl. Sci. Eng. 132, 16 (1999)

[16] Y. Terada et al., J. Nucl. Sci. Technol. 39(2), 413 (2002)

[17] I.H. Sarpün, J Fusion Energ. 34, 592-597 (2015)

[18] B. Demir et al., J Fusion Energ. 34, 808-816 (2015)

[19] I.H. Sarpün et al., J Fusion Energ. 34, 1306-1313 (2015)

[20] I.H. Sarpün et al., J Fusion Energ. 35, 725-729 (2016)

[21] Y. Han, Nucl. Phys. A780, 34 (2006)

[22] Z. Zhang et al., Ann. Nucl. Energ. 37, 130 (2010)

[23] J.-F. Duan et al., Commun. Theor. Phys. 54, 129 (2010)

[24] A. Aydin et al., J Fusion Energ. 32, 378 (2013)

[25] A. Aydin et al., Phys. Atom. Nucl. 77(3), 321 (2014)

[26] I.H. Sarpün et al., J Nucl. Sci. 1(1), 1 (2014)

[27] M. Drosg, B. Hoop, http://arxiv:1601.04956 (2014)

[28] M. Şahan et al., J Fusion Energ. 34, 493-499 (2015)

[29] H. Şahan et al., J Fusion Energ. 34, 16-23 (2015)

[30] M. Yiğit, E. Tel, Kerntechnik 79, 488-493 (2014)

[31] M. Yiğit, E. Tel, Nucl. Eng. Des. 280, 37-41 (2014)

[32] A. Kaplan, J Fusion Energ. 32, 382-388 (2013)

[33] A.J. Koning, M.C. Duijvestijn, Nucl. Phys. A744, 1576 (2004)

[34] A.J. Koning et al, TALYS-1.8, User Manual (NRG, The Netherlands, 2015)

[35] C. Kalbach, Phys. Rev. C 33, 818 (1986)

[36] W. Hauser, H. Feshbach, Phys. Rev. 87, 366 (1952)

[37] J. Raynal, Notes on ECIS84, CEA Saclay Report No. CEA-N-2772 (1994).

[38] S.M. Grimes et al., Nucl. Sci. and Eng. 62, 187, (1977)

[39] EXFOR Nuclear Library 